\title{
Optimality of the Users Strength in Optical PPM-CDMA Channels
}

\author{
Hossam M. H. Shalaby, Senior Member, IEEE
}

\begin{abstract}
An upper bound on the users strength in direct-detection optical code-division multiple-access communication systems, which employ pulse-position modulation techniques, is developed. This upper bound is consistent with the lower bound derived previously, as long as the transmitted information per photon is less than some positive threshold. The concept of users strength was introduced by the author in a previous paper, where it provides a measure to the maximum number of simultaneous users that can communicate simultaneously with arbitrary small probability of error.
\end{abstract}

Index Terms-Code-division multiple access (CDMA), direct-detection optical channel, optical code-division multiple access (OCDMA), pulse-position modulation (PPM).

\section{INTRODUCTION}

$\mathbf{O}$ PTICAL code-division multiple-access (OCDMA) communication systems have been given much interest in recent years [1]-[11]. In code-division multiple access (CDMA), multiplexing is achieved by assigning every user (in the same channel) a unique code sequence of length $L$ and weight $w$. These codes, called optical orthogonal codes (OOCs), must have good auto- and cross-correlation properties [2], [4]. Each user modulates a laser source before incorporating its signature code sequence. Both on-off keying (OOK) and pulse-position modulation (PPM) schemes can be used for source modulation. PPM has several advantages over OOK, where no threshold comparison is required at the receiver side, and its use of the laser energy is more efficient.

In optical PPM-CDMA systems, each user produces equiprobable $M$-ary data symbols. Each symbol $D$, which takes values from the finite set $\{0,1, \ldots, M-1\}$, modulates the position of a laser pulse of width $T_{c}$ within a time frame of duration $T$. That is, the laser pulse is signaled in position $i T / M$ if the data symbol $D=i, i \in\{0,1, \ldots, M-1\}$. The modulated laser pulse is then spread within a time slot of duration $\tau=T / M$ to generate the optical PPM-CDMA waveform. The spreading encoder is controlled by the signature code of the desired user. Optical pulses of duration $T_{c}$ each, represent the mark positions in the signature code. The time-slot duration must, in turn, satisfy the condition $\tau=L T_{c}$.

Paper approved by W. C. Kwong, the Editor for Optical Communications of the IEEE Communications Society. Manuscript received May 17, 2004; revised August 29, 2004 and September 20, 2004. This paper was presented in part at the International Conference on Information, Communications, and Signal Processing, Singapore, December, 1999.

The author is with the Department of Electrical Engineering, Faculty of Engineering, University of Alexandria, Alexandria 21544 Egypt (e-mail: shalaby@ieee.org).

Digital Object Identifier 10.1109/TCOMM.2004.841985
In [9], we have introduced the concept of the users strength. It provides a measure to the maximum number of users $N_{m}$ that can be accommodated simultaneously by the optical PPMCDMA system, while maintaining the error rate arbitrary small for any given positive value of the transmitted information per photon ( $\rho$ nats/photon). Indeed, we have shown that the dependence of $N_{m}$ on pulse-position multiplicity $M$ is not linear, and can be represented as

$$
\frac{N_{m}}{L}=M^{\theta}
$$

where $\theta$ denotes the users strength (to be defined rigorously in the next section). Our aim is to provide a complete characterization of $\theta$ so as to keep the error rate below a certain threshold $\epsilon$. In [9], we have only been able to provide lower bounds to the users strength. The tightness of these bounds has not been examined in [9].

The main objective in this paper is to develop an upper bound on the users strength, as defined in [9], to determine the tightness and optimality of the previous lower bound. The optimality of this lower bound is demonstrated by showing that any trial to increase the number of users above that estimated by the users strength's lower bound would lead to an error rate approaching one as $M \rightarrow \infty$. We are able to provide a complete characterization on the users strength as long as $\rho$ is less than some positive threshold. Above this threshold, however, we are unable to provide a good tight upper bound.

The statement of the problem is introduced in Section II, which is also devoted to some propositions and lemmas. The main results along with some discussions are given in Section III. Section IV provides the proof of the main theorem. Our conclusion is finally given in Section V.

\section{Problem Statement AND PReliminaries}

\section{A. Definitions}

Since we assume equiprobable data symbols in our study, the transmitted information in nats per channel use (nats per PPM symbol) is thus equal to $\log M$. This piece of information is denoted by $m$

$$
m \stackrel{\text { def }}{=} \log M \quad \text { nats/channel use. }
$$

The maximum number of simultaneous users and the probability of error are denoted by $N_{m}$ and $P_{m}[E]$, respectively. The subscript $m$ in both of them indicates that they depend on $M$. It might be omitted whenever there is no ambiguity in the sequel.

Given an OOC with length $L$, weight $w$, and auto- and crosscorrelation constraint $\lambda \in\{1,2 \ldots, w-1\}$, we have the following definitions. 
1) The Users Rate: We define the rate of the maximum number of simultaneous users (or simply the users rate) as $(1 / m) \log \left(N_{m} / L\right)$.

The motivation behind this definition is to introduce a normalized measure that is somehow robust for different CDMA systems. Indeed, the factor $N_{m} / L$ (number of users/chip) makes the definition robust against different CDMA-coding methods. In addition, to make it robust against different block-coding methods, we normalize the last quantity to a per-bit (or per-nat) basis. Since there are $\log _{2} M$ bits/symbol in an $M$-ary PPM block code, a useful and robust measure to the number of users is

$$
\frac{\log _{2} \frac{N_{m}}{L}}{\log _{2} M}=\frac{1}{m} \log \frac{N_{m}}{L} .
$$

2) An $\epsilon$-Achievable Users Rate: Given $0<\epsilon<1$, a nonnegative number $R$ is said to be $\epsilon$-achievable users rate for the PPM-CDMA optical channel, if for every $\delta>0$ and every sufficiently large $m$, we have

$$
\frac{1}{m} \log \frac{N_{m}}{L}>R-\delta \quad \text { with } \quad P_{m}[E] \leq \epsilon .
$$

3) An Achievable Users Rate: We say that $R>0$ is an achievable users rate if it is $\epsilon$-achievable for all $0<\epsilon<$ 1 .

4) The $\epsilon$-Users Strength: The supremum of $\epsilon$-achievable users rates is called the $\epsilon$-users strength $\theta(L, w, \lambda, \rho, \epsilon)$.

5) The Users Strength: The supremum of achievable users rates is called the users strength $\theta(L, w, \lambda, \rho)$.

In Appendix A, we show that the users strength is related to the $\epsilon$-users strength by

$$
\theta(L, w, \lambda, \rho)=\inf _{0<\epsilon<1} \theta(L, w, \lambda, \rho, \epsilon)=\lim _{\epsilon \rightarrow 0} \theta(L, w, \lambda, \rho, \epsilon) .
$$

From the above definitions, the importance of the users strength is obvious. It gives an estimate to the maximum number of users that can be accommodated in an optical PPM-CDMA network and still have an arbitrary small error rate. This estimate is given by

$$
\frac{N_{m}}{L}=M^{\theta(L, w, \lambda, \rho)-o(\epsilon)}
$$

where $o(\epsilon) \rightarrow 0$ as $\epsilon \rightarrow 0$. Further, it was shown in [9] that $P_{m}[E] \rightarrow 0$ as $M \rightarrow \infty$, which means that for large values of $M$, an estimate of the number of users can be given by $N_{m} / L \approx$ $M^{\theta(L, w, \lambda, \rho)}$.

In [9], we have developed simple lower bounds to the users strength defined above for any given $\rho \geq 0$. In the subsequent sections, we show (by deriving an upper bound) that this lower bound is tight within a finite interval of $\rho$.

\section{B. The Decision Rule}

The desired user collects the photons received within the mark positions of its signature code. This is done for every slot within the time frame. The number of slot with the largest count is declared to be the transmitted symbol. We denote the photon count collected in slot $i \in\{0,1, \ldots, M-1\}$ by $Y_{i}$. Symbol $i$ is thus declared to be the true one, if $Y_{i}>Y_{j}$ for every $j \neq i$. Hence, the probability of correct decision can be written as

$$
P_{m}[C]=\sum_{i=0}^{M-1} P_{m}[C \mid i] \operatorname{Pr}\{D=i\}=\frac{1}{M} \sum_{i=0}^{M-1} P_{m}[C \mid i]
$$

where

$$
P_{m}[C \mid i]=\operatorname{Pr}\left\{Y_{i}>Y_{j} \text {, every } j \neq i \mid D=i\right\} .
$$

In view of the symmetry of the channel, we can write

$$
P_{m}[C]=\operatorname{Pr}\left\{Y_{0}>Y_{j} \text {, every } j \neq 0 \mid D=0\right\} .
$$

\section{The Interference Probability}

We denote by $\kappa_{i t}, i \in\{0,1, \ldots, M-1\}, t \in\{1,2, \ldots, \lambda\}$, the number of other users that cause interference at $t$ pulse positions in slot $i$ of the desired user. Moreover, we denote the vector $\left(\kappa_{0 t}, \kappa_{1 t}, \ldots, \kappa_{M-1, t}\right)$ by $\kappa_{t}, t \in\{1,2, \ldots, \lambda\}$. The proofs of the following proposition and lemmas appear in Appendix A.

Proposition 1: In a chip-synchronous optical PPM-CDMA channel employing OOCs with weight $w>1$, length $L \geq w^{2}$, and auto- and cross-correlation constraint $\lambda<w$, if $P_{t}, t \in$ $\{1,2, \ldots, \lambda\}$, denotes the probability that a single user interferes with the desired user at $t$ pulse positions of one slot, then

$$
\sum_{t=1}^{\lambda} t \cdot P_{t}=\frac{w^{2}}{M L}
$$

where $M$ denotes the pulse-position multiplicity. Moreover, assuming that $P_{t}$ is uniform among different codeword pairs (which can be set as a restriction on the code construction), then

$$
\frac{1}{M L}<P_{\lambda} \leq \frac{w^{2}}{\lambda M L} .
$$

The equality is true when $\lambda=1$.

Lemma 1: If there are $N>1$ simultaneous users, and $P_{t}$, $t \in\{1,2, \ldots, \lambda\}$, is assumed to be uniform among different codeword pairs, then the random vector $\kappa_{t}, t \in\{1,2, \ldots, \lambda\}$, admits a multinomial distribution with parameters $N-1, P_{t}$

$$
\operatorname{Pr}\left\{\boldsymbol{\kappa}_{t}=\boldsymbol{l}_{t}\right\}=\frac{(N-1) !}{l_{0 t} ! l_{1 t} ! \cdots l_{M-1, t} ! s_{t} !} \cdot P_{t}^{N-1-s_{t}}\left(1-M P_{t}\right)^{s_{t}}
$$

where $\boldsymbol{l}_{t}$ is a realization vector for $\boldsymbol{\kappa}_{t}$, i.e., $\boldsymbol{l}_{t}=\left(l_{0 t}, l_{1 t}, \ldots, l_{M-1, t}\right)$ with $l_{i t} \geq 0 \forall i \in\{0,1, \ldots, M-1\}$ and $\sum_{i=0}^{M-1} l_{i t} \in\{0,1, \ldots, N-1\}$. Further

$$
s_{t} \stackrel{\text { def }}{=} N-1-\sum_{i=0}^{M-1} l_{i t} \geq 0 .
$$

Lemma 2: For any $i \in\{0,1, \ldots, M-1\}$, the random variables $\kappa_{i 1}, \kappa_{i 2}, \ldots, \kappa_{i \lambda}$ have a multinomial joint distribution with parameters $N-1, P_{1}, P_{2}, \ldots, P_{\lambda}$

$$
\begin{aligned}
\operatorname{Pr}\left\{\kappa_{i 1}=l_{i 1}, \ldots, \kappa_{i \lambda}=l_{i \lambda}\right\} & (N-1) ! \\
= & \frac{\left(N-1-\sum_{t=1}^{\lambda} l_{i t}\right) !}{l_{i 1} ! l_{i 2} ! \cdots l_{i \lambda} !\left(N-\sum_{t=1}^{\lambda} l_{i t}\right.} . \\
& \times P_{1}^{l_{i 1}} \ldots P_{\lambda}^{l_{i \lambda}}\left(1-\sum_{t=1}^{\left.\lambda-1-\sum_{t}\right)^{N}} .\right.
\end{aligned}
$$


Corollary 1: For any $i \in\{0,1, \ldots, M-1\}, t \in$ $\{1,2, \ldots, \lambda\}$, the random variable $\kappa_{i t}$ admits a binomial distribution with parameters $N-1, P_{t}$

$$
\operatorname{Pr}\left\{\kappa_{i t}=l_{i t}\right\}=\left(\begin{array}{c}
N-1 \\
l_{i t}
\end{array}\right) P_{t}^{l_{i t}}\left(1-P_{t}\right)^{N-1-l_{i t}} .
$$

Lemma 3: For any two integers $n \geq s \geq 0$

$$
\frac{n !}{s !} \leq 3 \sqrt{n-s+1} \cdot \frac{n^{n} e^{-(n-s)}}{s^{s}} .
$$

Lemma 4: For any $r \geq 0$ and $\nu \geq 1$

$$
\sum_{i=0}^{\nu-1} \frac{r^{i}}{i !} \leq \exp \left[r-\frac{r^{\nu}}{\nu !}+\frac{r^{\nu+1}}{\nu !}\right] \text {. }
$$

\section{MAIN RESUltS}

\section{A. Statements of the Results}

We demonstrate, in Theorem 1 and Corollary 2 below, the main results on the characterization of the users strength for the aforementioned system. The proof of the theorem is given in Section IV.

Theorem 1: In a chip-synchronous optical PPM-CDMA channel employing OOCs with weight $w>1$, length $L \geq w^{2}$, and auto- and cross-correlation constraint $\lambda<w$, the $\epsilon$-users strength is upper bounded by

$$
\theta(L, w, \lambda, \rho, \epsilon) \leq 1-\left\lceil\frac{w}{\lambda}\right\rceil^{-1}
$$

where $\epsilon \in(0,1)$ and $\lceil x\rceil$ denotes the smallest integer not less than $x$.

Corollary 2: In a chip-synchronous optical PPM-CDMA channel employing OOCs with weight $w>1$, length $L \geq w^{2}$, and auto- and cross-correlation constraint $\lambda<w$, if the photodetector statistics are Poisson, then the users strength is given by

$$
\theta(L, w, \lambda, \rho)=1-\left\lceil\frac{w}{\lambda}\right\rceil^{-1}
$$

where $\rho$ (the transmitted information in nats per photon) is constrained by

$$
\rho \leq \frac{\lambda\left\lceil\frac{w}{\lambda}\right\rceil}{\lambda\left\lceil\frac{w}{\lambda}\right\rceil-w+1}\left(\log \frac{w}{w-1}-\frac{1}{w}\right) .
$$

Proof: Immediate from Theorem 1 above, the lower bound from [9], and (1).

\section{B. Discussion of the Result}

Remark 1: The complete characterization for the users strength $\theta(L, w, \lambda, \rho)$ is given in Corollary 2. The characterization of the $\epsilon$-users strength $\theta(L, w, \lambda, \rho, \epsilon)$ is independent of $\epsilon$, $\epsilon \in(0,1)$, and must be equal to $\theta(L, w, \lambda, \rho)$. As we have mentioned earlier, our result holds for the Poisson system only when $\rho \leq(\lambda\lceil w / \lambda\rceil /(\lambda\lceil w / \lambda\rceil-w+1))(\log (w /(w-1))-(1 / w))$. Outside this interval, however, our characterization is incomplete where we are unable to provide a tight upper bound and only a lower bound on the $\epsilon$-users strength was shown in [9]. This problem is still open and more rigorous research is needed to come around it.

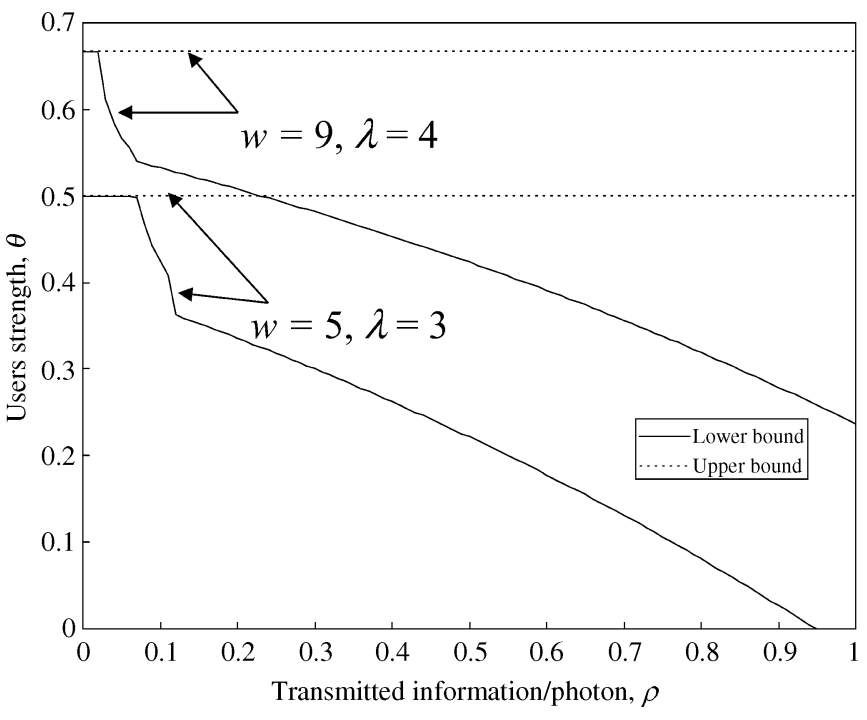

Fig. 1. Bounds on the users strength versus the transmitted information (nats per photon) for the Poisson system.

Remark 2: Both the upper bound from Theorem 1 and the lower bound from [9] are plotted in Fig. 1 for given system parameters. It can be seen that for $\rho$ smaller than some threshold, the users strength is constant and maximum. This means that the number of users cannot be increased above a certain limit. Otherwise, the multiple-user interference forces the error rate to be bounded away from zero. This observation indicates that the users strength is a sort of system-capacity measure. Indeed, given a CDMA code and a data rate in the optical PPM-CDMA channel, one cannot accommodate more users than that estimated by the maximum users strength, even if one is using unlimited, huge average power. Further, it can be seen from Fig. 1 that the region of maximum users strength shrinks as the code weight increases. This is because increasing the code weight will increase the rate of occurrence of mark hits among the users, and hence, more possible interference and more error rate. Thus, the number of users should be decreased to keep the error probability below the constraint. For convenience, we show in Table I how these regions scale with varying $w$ and $\lambda$.

Remark 3: Our complete characterization over some interval of $\rho$, as given in (3), is a little bit surprising. Indeed, its independence of $\rho$ over this interval implies that there is no tradeoff between the number of simultaneous users $N_{m} \approx L M^{\theta}$ and the average single-user power, $P_{\mathrm{av}}$

$$
P_{\mathrm{av}}=\frac{R_{T}}{\rho} \cdot \frac{h c}{\lambda_{0}}
$$

where $R_{T}$ is the data rate in nats/s, $h=6.626 \times 10^{-34} \mathrm{~J} . \mathrm{s}$ is Planck's constant, $c=3 \times 10^{8} \mathrm{~m} / \mathrm{s}$ is the speed of light, and $\lambda_{0}$ is the operating wavelength. That is, for fixed data rate and fixed error-rate constraint, we can decrease the average power (by increasing $\rho$ ) without disturbing the number of simultaneously communicating users (since $\theta$ is a constant here). This gives a great advantage in practice, e.g., we can have a very long optical-fiber link and allow the average power to decrease (because of loss, scattering, etc.), and still are able to accommodate the full number of users. Outside this interval, however, increasing $\rho$ (with fixed data rate and fixed error-rate constraint) 
TABLE I

REGION OF MAXIMUM USERS' STRENGTH

\begin{tabular}{|c|c|c|}
\hline $\begin{array}{l}\text { Code } \\
\text { weight }\end{array}$ & $\begin{array}{l}\text { Correlation } \\
\text { constraint }\end{array}$ & $\begin{array}{l}\text { Threshold } \\
\text { (nats/photon) }\end{array}$ \\
\hline \multirow[t]{2}{*}{3} & 1 & 0.216395 \\
\hline & 2 & 0.144264 \\
\hline \multirow[t]{4}{*}{5} & 1 & 0.115718 \\
\hline & 2 & 0.069431 \\
\hline & 3 & 0.069431 \\
\hline & 4 & 0.046287 \\
\hline \multirow[t]{6}{*}{7} & 1 & 0.079055 \\
\hline & 2 & 0.045174 \\
\hline & 3 & 0.033881 \\
\hline & 4 & 0.045174 \\
\hline & 5 & 0.028234 \\
\hline & 6 & 0.022587 \\
\hline \multirow[t]{8}{*}{9} & 1 & 0.060047 \\
\hline & 2 & 0.033360 \\
\hline & 3 & 0.060047 \\
\hline & 4 & 0.020016 \\
\hline & 5 & 0.033360 \\
\hline & 6 & 0.020016 \\
\hline & 7 & 0.015568 \\
\hline & 8 & 0.013344 \\
\hline
\end{tabular}

would decrease the number of simultaneous users (since $\theta$ decreases and $N_{m} \approx L M^{\theta}$ ), and the above advantage will be lost. The reason is that for large values of $\rho$, the average power becomes too small to tolerate the shot-noise effect at the receiver. We wish to emphasize here that the region of constant users strength given in (3) corresponds to a practical range of average power. For example, let $R_{T}=1 \mathrm{~Gb} / \mathrm{s}, w=5, \lambda=3$, and $\lambda_{0}=1.55 \mu \mathrm{m}$. From Fig. 1 or (3), this region is given by $\rho \leq 0.069$ nats/photon, and the corresponding region of average power is thus $P_{\mathrm{av}} \geq 1.85 \mathrm{nW}$. Further, this restricted range on $\rho$ gives an acceptable probability of error. Indeed, the corresponding region of average photons/nat (for the last example) is $\mu=1 / \rho \geq 14.49$ photons/nat, which is wide enough. By consulting [7] and Table I, it can be seen that good error rate can be achieved with suitable choices of both $\rho$ and $M$.

Remark 4: The result of the theorem indicates that for given code parameters, the numbers of simultaneous users can be increased freely by increasing the pulse-position multiplicity $M$. Indeed, this is obvious from the positivity of the users strength. Although the number of available codewords will be limited by code parameters, it has been proposed in [11] that it is possible to assign a given codeword to more than one user, provided that a suitable protocol is adopted by users. Of course, there is a tradeoff between $M$ and both the data rate $R_{T}$ and peak power $P_{p}$

$$
R_{T}=\frac{\log M}{M L T_{c}} \text { nats/s, } \quad P_{p}=P_{\mathrm{av}} \frac{M L}{w}
$$

Indeed, the price to be paid when increasing $M$ is an increase in the peak power and a decrease in the data rate. Alternatively, in order to keep the data rate unchanged, there should be an increase in the bandwidth required for optical pulses [7]. In practice, this increase in bandwidth in addition to both fiber dispersion and photodetector's rise time set a physical limitation that restricts the actual number of users sharing the channel.

\section{Proof of TheOREM 1}

Remark 5: Since the system with ideal photodiodes provides an upper bound to the number of users of systems with Poisson photodiodes, it suffices to prove the above theorem for the ideal case. We show that increasing the $\epsilon$-users strength beyond $1-$ $\lceil w / \lambda\rceil^{-1}$ leads to an error probability which converges to one as $m$ goes to $\infty$.

Remark 6: In our derivation of this theorem, we will show that if $\left(N_{m} / L\right)>M^{1-\lceil w / \lambda\rceil^{-1}+\delta}, \delta>0$, then the correct probability $P_{m}[C]$ goes to zero with $M \rightarrow \infty$. The proof will be done under the assumption that $\lim _{M \rightarrow \infty}\left(N_{m} / M L\right)=0$, which is true for $N_{m}$, satisfying

$$
M^{1-\left\lceil\frac{w}{\lambda}\right\rceil^{-1}+\delta}<\frac{N_{m}}{L} \leq M^{1-\xi}
$$

any $0<\delta<\lceil w / \lambda\rceil^{-1}$, and $0<\xi<\lceil w / \lambda\rceil^{-1}-\delta$. Increasing $N_{m}$ above the aforementioned interval should lead to a worse correct probability because the multiple-access interference would increase. Hence, even if $\left(N_{m} / L\right) \geq M^{1-\xi}$, we will get $P_{m}[C] \rightarrow 0$ as $m \rightarrow \infty$. This argument justifies that our assumption can be removed without disturbing the theorem.

Remark 7: Our proof of this theorem is based on chip-synchronous assumption. This assumption is valid for synchronous OCDMA systems [5], [7]. For asynchronous systems, however, this assumption can be considered as an approximation to the strong chip-asynchronous interference pattern (which is a special case of the generalized interference pattern) [3].

Let $\nu \stackrel{\text { def }}{=}\lceil w / \lambda\rceil$. Assume by contradiction that $\left(N_{m} / L\right)>$ $\exp [m(1-(1 / \nu)+\delta)]$, with $0<\delta<1 / \nu$. We will show that this leads to a correct probability that converges to zero. We denote by $I_{j}, j \in\{0,1, \ldots, M-1\}$, the number of interfering pulses in slot $j$. The correct probability in (2) can now be estimated as

$$
\begin{aligned}
P_{m}[C]= & \operatorname{Pr}\left\{Y_{0}>Y_{j}, \text { every } j \neq 0 \mid D=0\right\} \\
= & \operatorname{Pr}\left\{w+I_{0}>I_{j} \forall j \geq 1\right\} \\
= & \sum_{i_{0}} \operatorname{Pr}\left\{I_{j}<w+i_{0} \forall j \geq 1 \mid I_{0}=i_{0}\right\} \operatorname{Pr}\left\{I_{0}=i_{0}\right\} \\
\leq & \operatorname{Pr}\left\{I_{j}<w \forall j \geq 1 \mid I_{0}=0\right\} \operatorname{Pr}\left\{I_{0}=0\right\} \\
& +\operatorname{Pr}\left\{I_{0} \neq 0\right\} .
\end{aligned}
$$

But using the Markov inequality, Corollary 1, and Proposition 1 , we get

$$
\begin{aligned}
\operatorname{Pr}\left\{I_{0} \neq 0\right\} & =\operatorname{Pr}\left\{\sum_{t=1}^{\lambda} t \cdot \kappa_{0 t} \geq 1\right\} \leq E\left\{\sum_{t=1}^{\lambda} t \cdot \kappa_{0 t}\right\} \\
& =(N-1) \sum_{t=1}^{\lambda} t \cdot P_{t}=\frac{w^{2}(N-1)}{M L}
\end{aligned}
$$


where $E\{\cdot\}$ denotes the expectation value. Thus, $\operatorname{Pr}\left\{I_{0} \neq 0\right\}$ converges to 0 as $m \rightarrow \infty$. Hence, it suffices to show that

$P_{m}\left[C, I_{0}=0 \mid D=0\right] \stackrel{\text { def }}{=} \operatorname{Pr}\left\{I_{j}<w \forall j \geq 1 \mid I_{0}=0\right\} \operatorname{Pr}\left\{I_{0}=0\right\}$ diminishes to zero as $m \rightarrow \infty$. Notice that

$$
\begin{aligned}
P_{m} & {\left[C, I_{0}=0 \mid D=0\right] } \\
& =\operatorname{Pr}\left\{\sum_{t=1}^{\lambda} t \cdot \kappa_{j t}<w \forall j \geq 1, \kappa_{0 t}=0, t \in\{1,2, \ldots, \lambda\}\right\} \\
& \leq \operatorname{Pr}\left\{\lambda \kappa_{j \lambda}<w \forall j \geq 1, \kappa_{0 \lambda}=0\right\} \\
& =\operatorname{Pr}\left\{\kappa_{j \lambda}<\nu \forall j \geq 1, \kappa_{0 \lambda}=0\right\} .
\end{aligned}
$$

Define the set

$$
\chi \stackrel{\text { def }}{=}\{0,1, \ldots, \nu-1\} .
$$

Denoting the vector $\left(l_{1 \lambda}, l_{2 \lambda}, \ldots, l_{M-1, \lambda}\right)$ by $l_{1}^{M-1}$, the last probability can be rewritten as

$$
\begin{aligned}
& P_{m} {\left[C, I_{0}=0 \mid D=0\right] } \\
& \leq \sum_{\substack{l_{1}^{M-1} \in \chi^{M-1}: \\
\sum_{i=1}^{M-1} l_{i \lambda} \leq N-1}} \operatorname{Pr}\left\{\kappa_{\lambda}=\left(0, l_{1 \lambda}, l_{2 \lambda}, \ldots, l_{M-1, \lambda}\right)\right\} \\
&=\sum_{\substack{Q \in \mathcal{P}_{M-1}(\chi): Q=\left(\frac{j_{0}}{M-1}, \ldots, \frac{j_{\nu-1}}{M-1}\right), \quad \sum_{i=1}^{\nu-1}{ }_{i j_{i} \leq N-1}, \sum_{i=0}^{\nu-1} j_{i}=M-1}} P\left(T_{Q}^{M-1} \mid \kappa_{0 \lambda}=0\right) \\
& \quad \times \operatorname{Pr}\left\{\kappa_{0 \lambda}=0\right\} .
\end{aligned}
$$

Here $T_{Q}^{M-1}$ [12] denotes the set of sequences of type $Q=$ $\left(j_{0} /(M-1), \ldots,\left(j_{\nu-1}\right) /(M-1)\right)$ in $\mathcal{X}^{M-1}$, where, for any $i \in \mathcal{X}, j_{i}$ denotes the number of elements in $l_{1}^{M-1} \in T_{Q}^{M-1}$ equal to $i$, and $\mathcal{P}_{M-1}(\mathcal{X})$ denotes the set of all types of sequences in $\mathcal{X}^{M-1}$ [12]. The last probability can further be upper bounded as

$$
\begin{aligned}
& P_{m}\left[C, I_{0}=0 \mid D=0\right] \leq\left|\mathcal{P}_{M-1}(\chi)\right| \max _{Q \in \mathcal{P}_{M-1}(\chi): Q=\left(\frac{j_{0}}{M-1}, \ldots, \frac{j_{\nu-1}}{M-1}\right),} \\
& \times P\left(T_{Q}^{M-1} \mid \kappa_{0 \lambda}^{\nu-1}{ }_{i=1}=0\right) \operatorname{Pr}\left\{\kappa_{i} \leq N-1, \sum_{i=0}^{\nu-1}{ }_{j i}=M-1\right. \\
&\left.\kappa_{0 \lambda}=0\right\}
\end{aligned}
$$

where $\left|\mathcal{P}_{M-1}(\mathcal{X})\right|$ denotes the size of $\mathcal{P}_{M-1}(\mathcal{X})$. Next, we develop an estimate to the last probability as follows. For any $Q=\left(j_{0} /(M-1), \ldots,\left(j_{\nu-1}\right) /(M-1)\right)$, define

$$
\begin{aligned}
P_{T} \stackrel{\text { def }}{=} & P\left(T_{Q}^{M-1} \mid \kappa_{0 \lambda}=0\right) \operatorname{Pr}\left\{\kappa_{0 \lambda}=0\right\} \\
= & \left|T_{Q}^{M-1}\right| \operatorname{Pr}\left\{\kappa_{\lambda}=\left(0, l_{1 \lambda}, l_{2 \lambda}, \ldots, l_{M-1, \lambda}\right)\right\} \\
= & \frac{(N-1) !}{s ! 2 !^{j_{2}} 3 !^{j_{3}} \ldots(\nu-1) !^{j_{\nu-1}}} \cdot P_{\lambda}^{N-1-s}\left(1-M P_{\lambda}\right)^{s} \\
& \cdot \frac{(M-1) !}{j_{0} ! j_{1} ! \ldots j_{\nu-1} !}
\end{aligned}
$$

where

$$
s=N-1-\sum_{i=1}^{\nu-1} i j_{i}, \quad j_{0}=M-1-\sum_{i=1}^{\nu-1} j_{i} .
$$

Using Lemma 3 and the fact that [12]

$$
\frac{(M-1) !}{j_{0} ! j_{1} ! \ldots j_{\nu-1} !}=\left|T_{Q}^{M-1}\right| \leq \exp [(M-1) H(Q)]
$$

where $H(Q)$ is the usual informational entropy function, we can upper bound $P_{T}$ as

$$
\begin{aligned}
P_{T} \leq 3 \sqrt{N-s} & \frac{\left(\frac{(N-1)}{e}\right)^{N-1}}{\left(\frac{s}{e}\right)^{s} 2 ! j_{2} 3 ! j_{3} \ldots(\nu-1) ! j_{\nu-1}} \\
& \cdot P_{\lambda}^{N-1-s}\left(1-M P_{\lambda}\right)^{s} \cdot \exp [(M-1) H(Q)] .
\end{aligned}
$$

Taking the logarithm of $P_{T}$ yields

$$
\begin{aligned}
\log P_{T} \leq & -\sum_{i=1}^{\nu-1} j_{i} \log \frac{j_{i}}{M-1} \\
& -\left(M-1-\sum_{i=1}^{\nu-1} j_{i}\right) \log \frac{M-1-\sum_{i=1}^{\nu-1} j_{i}}{M-1} \\
& +\sum_{i=1}^{\nu-1} i j_{i} \log \frac{P_{\lambda}}{1-M P_{\lambda}} \\
& -\sum_{i=1}^{\nu-1} j_{i} \log i !+(N-1) \log \frac{N-1}{e} \\
& -\left(N-1-\sum_{i=1}^{\nu-1} i j_{i}\right) \log \frac{N-1-\sum_{i=1}^{\nu-1} i j_{i}}{e} \\
& +(N-1) \log \left(1-M P_{\lambda}\right)+\log (3 \sqrt{N-s}) .
\end{aligned}
$$

Now we maximize $\log P_{T}$ subject to the constraints $s \geq 0$ and $j_{i} \geq 0$. It is not a difficult task to check that these constraints are inactive. Thus, the necessary conditions for optimization are

$$
(\forall i \in \chi) \quad \frac{\partial}{\partial j_{i}} \log P_{T}=0 .
$$

This yields

$$
\log \frac{j_{0} s^{i} P_{\lambda}^{i}}{j_{i}\left(1-M P_{\lambda}\right)^{i} i !}=0
$$

Or for any $i \in \mathcal{X}$

$$
j_{i}=\frac{j_{0}}{i !}\left(\frac{s P_{\lambda}}{1-M P_{\lambda}}\right)^{i}=\frac{j_{0}}{i !} r^{i}
$$

where

$$
r \stackrel{\text { def }}{=} \frac{s P_{\lambda}}{1-M P_{\lambda}}=i \frac{j_{i}}{j_{i-1}} .
$$

Substituting in (5) yields

$$
\begin{aligned}
\log P_{T} & \leq \log 3 \sqrt{N-s}+(M-1) \log \frac{M-1}{j_{0}} \\
& +(N-1) \log \frac{(N-1)\left(1-M P_{\lambda}\right)}{s}-(N-1-s) .
\end{aligned}
$$

Notice that

$$
\begin{aligned}
(M-1) \log \frac{M-1}{j_{0}} & =(M-1) \log \frac{\sum_{i=0}^{\nu-1} j_{i}}{j_{0}} \\
& =(M-1) \log \sum_{i=0}^{\nu-1} \frac{r^{i}}{i !} \\
& \leq(M-1)\left(r-\frac{r^{\nu}}{\nu !}+\frac{r^{\nu+1}}{\nu !}\right)
\end{aligned}
$$


where the last equality follows from (6) and the last inequality from Lemma 4. Next we develop estimates for both $r$ and $s$. From (7), we may write

$$
r=\frac{j_{1}+2 j_{2}+\cdots+(\nu-1) j_{\nu-1}}{j_{0}+j_{1}+\cdots+j_{\nu-2}}=\frac{N-1-s}{M-1-j_{\nu-1}} .
$$

Substituting back to (7) and solving for $s$

$$
s=\frac{(N-1)\left(1-M P_{\lambda}\right)}{1-P_{\lambda}\left(1+j_{\nu-1}\right)}
$$

Using $s$ from (10) in (7) yields

$$
r=\frac{(N-1) P_{\lambda}}{1-P_{\lambda}\left(1+j_{\nu-1}\right)}
$$

Substituting (9) and (10) in (8), we get

$$
\begin{aligned}
\log P_{T} \leq & \log 3 \sqrt{N-s}+(M-1)\left(r-\frac{r^{\nu}}{\nu !}+\frac{r^{\nu+1}}{\nu !}\right) \\
& +(N-1) \log \left[1-P_{\lambda}\left(1+j_{\nu-1}\right)\right]-(N-1-s) \\
\leq & \log 3 \sqrt{N-s}+(M-1)\left(r-\frac{r^{\nu}}{\nu !}+\frac{r^{\nu+1}}{\nu !}\right) \\
& -(N-1) P_{\lambda}\left(1+j_{\nu-1}\right)-(N-1-s) .
\end{aligned}
$$

After some algebraic manipulation involving (10) and (11), we obtain

$$
\begin{aligned}
\log P_{T} \leq \log 3 \sqrt{N-s}-(M-1) & \left.\frac{r^{\nu}}{\nu !}-\frac{r^{\nu+1}}{\nu !}\right) \\
& -r+r P_{\lambda}\left(1+j_{\nu-1}\right)^{2} .
\end{aligned}
$$

For large values of $M$, the last two terms can further be bounded as follows:

$$
\begin{aligned}
-r+r P_{\lambda}\left(1+j_{\nu-1}\right)^{2} & \leq r P_{\lambda} j_{\nu-1}^{2} \leq r P_{\lambda} j_{0}^{2} \frac{r^{2 \nu-2}}{(\nu-1) !^{2}} \\
& \leq \frac{w^{2}}{\lambda M L}(M-1)^{2} \frac{r^{2 \nu-1}}{(\nu-1) !^{2}} \\
& \leq \frac{w^{2}}{(\nu-1) !^{2}} \frac{(M-1) r^{2 \nu-1}}{\lambda L}
\end{aligned}
$$

Substituting back would yield

$\log P_{T} \leq \log 3 \sqrt{N-s}-(M-1) \frac{r^{\nu}}{\nu !}\left(1-r-r^{\nu-1} \frac{w^{2} \nu}{(\nu-1) ! \lambda L}\right)$

Now we bound the ratio $r$ as follows. Since $s \geq 0$ and $j_{i} \geq 0$, then $N-1 \geq(\nu-1) j_{\nu-1}$. Hence

$$
\begin{aligned}
& r=\frac{(N-1) P_{\lambda}}{1-P_{\lambda}\left(1+j_{\nu-1}\right)} \geq(N-1) P_{\lambda} \\
& r \leq \frac{(N-1) P_{\lambda}}{1-P_{\lambda}\left(1+\frac{N-1}{\nu-1}\right)} \leq \frac{N-1}{\lambda M \frac{L}{w^{2}}-1-\frac{N-1}{\nu-1}}
\end{aligned}
$$

Thus, $r \rightarrow 0$ as $M \rightarrow \infty$. Using (10), (11), and (12) in (4) gives

$$
\begin{aligned}
\log & P_{m}\left[C, I_{0}=0 \mid D=0\right] \\
\leq & \log \left|\mathcal{P}_{M-1}(\chi)\right|+\max \log P_{T} \leq w \log M+\max \log P_{T} \\
\leq & w \log M+\log 3 \sqrt{\left(M-1-j_{\nu-1}\right) r+1} \\
& -(M-1) \frac{r^{\nu}}{\nu !}\left(1-r-r^{\nu-1} \frac{w^{2} \nu}{(\nu-1) ! \lambda L}\right)
\end{aligned}
$$

where we have invoked the type-counting lemma [12] to justify the inequality $\left|\mathcal{P}_{M-1}(\mathcal{X})\right| \leq M^{w}$. For $M$ large enough, $1-r-$ $r^{\nu-1}\left(w^{2} \nu /(\nu-1) ! \lambda L\right)$ converges to one, since $r \rightarrow 0$. Thus, including the lower bound in (13a), we conclude

$$
\begin{aligned}
& \log P_{m}\left[C, I_{0}=0 \mid D=0\right] \\
& \leq w \log M+\log 3 \sqrt{M r+1}-(M-1) \frac{r^{\nu}}{\nu !}\left(1-\xi_{m}\right) \\
& \leq w \log M+\log 3 \sqrt{M r+1}-(M-1)\left((N-1) P_{\lambda}\right)^{\nu} \cdot \frac{1-\xi_{m}}{\nu !}
\end{aligned}
$$

where $\xi_{m} \rightarrow 0$ as $M \rightarrow \infty$. Finally, we notice that

$$
\frac{N}{L}>\exp \left[m\left(1-\frac{1}{\nu}+\delta\right)\right]=M^{1-\frac{1}{\nu}+\delta}
$$

yields

$$
\begin{aligned}
P_{m}\left[C, I_{0}=0 \mid D=0\right] & \leq \exp \left[-\left(M P_{\lambda} L\right)^{\nu} M^{\delta \nu} \frac{1-2 \xi_{m}}{\nu !}\right] \\
& <\exp \left[-M^{\delta \nu} \frac{1-2 \xi_{m}}{\nu !}\right] \rightarrow 0 .
\end{aligned}
$$

The last inequality is justified by invoking Proposition 1.

\section{CONCLUSION}

In this paper, we have been able to find an upper bound to the users strength, introduced in [9], for direct-detection optical PPM-CDMA channels. Combining this upper bound with the lower bound of [9] provides a complete characterization of the users strength whenever the transmitted information per photon is below a certain threshold. Above this threshold, however, our characterization is only partial (the upper bound is not tight enough.) It has also been shown that within the aforementioned interval, the users strength is independent of $\rho$, and the number of users do not increase by reducing $\rho$.

APPENDIX A

\section{PROOFS OF PROPOSITIONS AND LEMMAS}

\section{A. Proof of Equation (1)}

To prove this assertion, we proceed as follows. Given $0<$ $\epsilon<1$, we define

$\mathcal{A}_{\epsilon} \stackrel{\text { def }}{=}\{R: \forall \delta>0, \exists \mathcal{M}$ such that $\forall m \geq \mathcal{M}$,

$$
\left.\frac{1}{m} \log \frac{N_{m}}{L}>R-\delta \text { with } P_{m}[E] \leq \epsilon\right\} \text {. }
$$


Thus from Definition 4, we have

$$
\theta(L, w, \lambda, \rho, \epsilon)=\sup _{R \in \mathcal{A}_{\epsilon}} R
$$

Furthermore, let

$$
\mathcal{A} \stackrel{\text { def }}{=} \bigcap_{0<\epsilon<1} \mathcal{A}_{\epsilon}
$$

Thus from Definition 5, we have

$$
\theta(L, w, \lambda, \rho)=\sup _{R \in \mathcal{A}} R
$$

For any $0<\epsilon<1$, since $\mathcal{A} \subset \mathcal{A}_{\epsilon}$, we get $\theta(L, w, \lambda, \rho) \leq$ $\sup _{R \in \mathcal{A}_{\epsilon}} R=\theta(L, w, \lambda, \rho, \epsilon)$, or

$$
\theta(L, w, \lambda, \rho) \leq \inf _{0<\epsilon<1} \theta(L, w, \lambda, \rho, \epsilon) .
$$

On the other hand, it is easy to check that for any $0<\epsilon_{1}<\epsilon_{2}<$ $1, \mathcal{A}_{\epsilon_{1}} \subset \mathcal{A}_{\epsilon_{2}}$ (which also implies that $\theta(L, w, \lambda, \rho, \epsilon)$ decreases as $\epsilon$ decreases). Hence, $\mathcal{A}=\lim _{\epsilon \rightarrow 0} \mathcal{A}_{\epsilon}$ and $\exists \epsilon_{0}, 0<\epsilon_{0}<1$, such that

$$
\begin{aligned}
\theta(L, w, \lambda, \rho) & \geq \sup _{R \in \mathcal{A}_{\epsilon_{0}}} R=\theta\left(L, w, \lambda, \rho, \epsilon_{0}\right) \\
& \geq \inf _{0<\epsilon<1} \theta(L, w, \lambda, \rho, \epsilon) .
\end{aligned}
$$

From the above discussion, we conclude that

$$
\theta(L, w, \lambda, \rho)=\inf _{0<\epsilon<1} \theta(L, w, \lambda, \rho, \epsilon) .
$$

Since $\theta(L, w, \lambda, \rho, \epsilon)$ decreases as $\epsilon$ decreases, the last infimum is achieved when $\epsilon \rightarrow 0$, and this completes the proof of (1).

\section{B. Proof of Proposition 1}

The proof of the first assertion appears in [9]. The upper bound in the second assertion is immediate from the first one, since

$$
\frac{w^{2}}{M L}=\sum_{t=1}^{\lambda} t \cdot P_{t} \geq \lambda \cdot P_{\lambda} .
$$

The equality is true when $\lambda=1$. As for the lower bound, we notice that

$$
\begin{aligned}
P_{\lambda}= & \frac{1}{M} \cdot \operatorname{Pr}\{\text { exactly } \lambda \text { hits occur in one slot under } \\
= & \left.\frac{1}{M} \quad L \text { shifts }\right\} \\
& \cdot \frac{\text { number of times } \lambda \text { hits may occur under } L \text { shifts }}{L} \\
\geq & \frac{1}{M L} .
\end{aligned}
$$

The above inequality is true, since the cross-correlation constraint equals $\lambda$, which ensures the occurrence of at least one mark hit under $L$ shifts. The equality is true when $w=\lambda=1$. Since we use the assumption $w>\lambda \geq 1$, this equality cannot hold, and we have $P_{\lambda}>1 / M L$.

\section{Proof of Lemma 1}

Fix $t \in\{1,2, \ldots, \lambda\}$. Let $A_{i}, i \in\{0,1, \ldots, M-1\}$, be the event that a single user interferes with the desired user at $t$ pulse positions within slot $i$. Each of these disjoint events occurs with probability $P_{t}$. Further, let $A_{M}$ be the event that a single user interferes with the desired user at $q$ pulse positions, $q \in\{0,1, \ldots, t-1, t+1, \ldots, \lambda\}$. Obviously, $A_{M}$ occurs with probability $1-M P_{t}$. Thus, $l_{i t}, i \in\{0,1, \ldots, M-1\}$ corresponds to the number of times that $A_{i}$ occurs, and $s_{t}$ corresponds to the number of times that $A_{M}$ occurs. Moreover, $s_{t}+\sum_{i=0}^{M-1} l_{i t}=N-1$. Hence, this formulation leads to the well-known multinomial distribution.

\section{Proof of Lemma 2}

Similar to that of Lemma 1.

\section{E. Proof of Corollary 1}

Immediate from any of Lemmas 1 or 2.

\section{F. Proof of Lemma 3}

The Robbins' sharpening of Stirling's formula is given by

$$
\sqrt{2 \pi} k^{k+\frac{1}{2}} e^{-k+\frac{1}{12(k+1)}} \leq k ! \leq \sqrt{2 \pi} k^{k+\frac{1}{2}} e^{-k+\frac{1}{12 k}} .
$$

For any $k \geq 1$, the last inequality can further be increased as

$$
k ! \leq 3 \sqrt{k}\left(\frac{k}{e}\right)^{k} \leq 3 \sqrt{k+1}\left(\frac{k}{e}\right)^{k}
$$

Moreover, this inequality remains true for $k=0$ as well. Indeed, in this case, $k !=1 \leq \lim _{k \rightarrow 0} 3 \sqrt{k+1}(k / e)^{k}=3$. Now consider

$$
\begin{aligned}
\frac{n !}{s !} & =\left(\begin{array}{c}
n \\
s
\end{array}\right)(n-s) ! \leq \exp \left[n h\left(\frac{s}{n}\right)\right](n-s) ! \\
& \leq 3 \sqrt{n-s+1}\left(\frac{n-s}{e}\right)^{n-s} \exp \left[n h\left(\frac{s}{n}\right)\right]
\end{aligned}
$$

where $h(p) \stackrel{\text { def }}{=}-p \log p-(1-p) \log (1-p)$ is the binary informational entropy function. Substituting for $h(s / n)$ would conclude the required upper bound. 


\section{G. Proof of Lemma 4}

$$
\begin{aligned}
\text { Right-hand side } & =e^{r} \cdot e^{-\frac{r^{\nu}}{\nu !}+\frac{r^{\nu+1}}{\nu !}} \\
& \geq\left(\sum_{i=0}^{\nu} \frac{r^{i}}{i !}\right)\left(1-\frac{r^{\nu}}{\nu !}+\frac{r^{\nu+1}}{\nu !}\right) \\
& =\sum_{i=0}^{\nu} \frac{r^{i}}{i !}-\frac{r^{\nu}}{\nu !} \sum_{i=0}^{\nu} \frac{r^{i}}{i !}+\frac{r^{\nu+1}}{\nu !} \sum_{i=0}^{\nu} \frac{r^{i}}{i !} \\
& =\sum_{i=0}^{\nu-1} \frac{r^{i}}{i !}-\frac{r^{\nu}}{\nu !} \sum_{i=1}^{\nu} \frac{r^{i}}{i !}+\frac{r^{\nu+1}}{\nu !} \sum_{i=0}^{\nu} \frac{r^{i}}{i !} \\
& \geq \sum_{i=0}^{\nu-1} \frac{r^{i}}{i !}=\text { Left-hand side. }
\end{aligned}
$$

The last inequality is true, because we can write

$$
\sum_{i=1}^{\nu} \frac{r^{i}}{i !} \leq \sum_{i=1}^{\nu} \frac{r^{i}}{(i-1) !}=\sum_{k=0}^{\nu-1} \frac{r^{k+1}}{k !} \leq r \sum_{i=0}^{\nu} \frac{r^{i}}{i !} .
$$

\section{REFERENCES}

[1] P. R. Prucnal, M. A. Santoro, and T. R. Fan, "Spread spectrum fiber-optic local area network using optical processing," IEEE/OSA J. Lightwave Technol., vol. LT-4, pp. 547-554, May 1986.

[2] F. R. K. Chung, J. A. Salehi, and V. K. Wei, "Optical orthogonal codes: Design, analysis, and applications," IEEE Trans. Inf. Theory, vol. 35, pp. 595-604, May 1989.

[3] J. A. Salehi, "Code division multiple-access techniques in optical fiber networks - Part I: Fundamental principles," IEEE Trans. Commun., vol. 37, pp. 824-833, Aug. 1989.

[4] H. Chung and P. V. Kumar, "Optical orthogonal codes—New bounds and an optimal construction," IEEE Trans. Inf. Theory, vol. 36, pp. 866-873, Jul. 1990.

[5] W. C. Kwong, P. A. Perrier, and P. R. Prucnal, "Performance comparison of asynchronous and synchronous code-division multiple access," IEEE Trans. Commun., vol. 39, pp. 1625-1634, Nov. 1991.

[6] H. M. Kwon, "Optical orthogonal code-division multiple-access system-Part II: Multibits/sequence-period OOCDMA," IEEE Trans. Commun., vol. 42, pp. 2592-2599, Aug. 1994.

[7] H. M. H. Shalaby, "Performance analysis of optical synchronous CDMA communication systems with PPM signaling," IEEE Trans. Commun., vol. COM-43, pp. 624-634, Feb.-Apr. 1995.

[8] T. Ohtsuki, "Performance analysis of direct-detection optical asynchronous CDMA systems with double optical hard-limiters," IEEE/OSA J. Lightwave Technol., vol. 15, pp. 452-457, Mar. 1997.
[9] H. M. H. Shalaby, "Maximum achievable number of users in optical PPM-CDMA local area networks," IEEE/OSA J. Lightwave Technol., vol. 18, pp. 1187-1196, Sep. 2000.

[10] S. Zahedi and J. A. Salehi, "Analytical comparison of various fiber-optic CDMA receiver structures," IEEE/OSA J. Lightwave Technol., vol. 18, pp. 1718-1727, Dec. 2000.

[11] H. M. H. Shalaby, "Optical CDMA random access protocols with and without pretransmission coordination," IEEE/OSA J. Lightwave Technol., vol. 21, pp. 2455-2462, Nov. 2003.

[12] I. Csiszár and J. Körner, Information Theory: Coding Theorems for Discrete Memoryless Systems. New York: Academic, 1982.

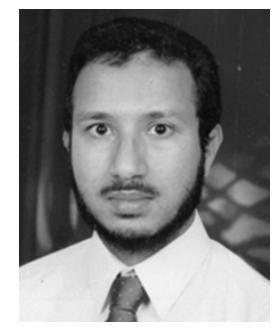

Hossam M. H. Shalaby (S'83-M'91-SM'99) was born in Giza, Egypt, in 1961. He received the B.S. and M.S. degrees from the University of Alexandria, Alexandria, Egypt, in 1983 and 1986, respectively, and the Ph.D. degree from the University of Maryland, College Park, in 1991, all in electrical engineering.

In 1991, he joined the Department of Electrical Engineering, University of Alexandria, as an Assistant Professor. He was promoted to the position of Associate Professor in 1996, and then to Professor in 2001 (current position). Since December 2000, he has been an Adjunct Professor with the Department of Electrical and Information Engineering, Faculty of Sciences and Engineering, Laval University, Quebec, QC, Canada. From March to April 1996, he was a Visiting Professor at the Electrical Engineering Department, Beirut Arab University, Beirut, Lebanon. From September 1996 to January 1998, he was an Associate Professor with the Electrical and Computer Engineering Department, International Islamic University Malaysia, Kuala Lumpur, Malaysia, and from February 1998 to December 1998, he was with the School of Electrical and Electronic Engineering, Nanyang Technological University, Singapore, where he was a Senior Lecturer, and from January 1999 to February 2001, an Associate Professor. His research interests include optical communications, optical CDMA, spread-spectrum communications, and information theory.

Dr. Shalaby received the SRC fellowship from 1987 to 1991 from the Systems Research Center, MD, the Shoman Prize for Young Arab Researchers in 2002 from the Abdul Hameed Shoman Foundation, Amman, Jordan, the State Award twice in 1995 and 2001 from the Academy of Scientific Research and Technology, Egypt, the University Award in 1996 from the University of Alexandria, and the Soliman Abd-El-Hay Award in 1995 from the Academy of Scientific Research and Technology, Egypt. He has served as a Student Branch Counselor for Alexandria University, IEEE Alexandria and North Delta Subsection, since 2002, and served as a Chairman of the Student Activities Committee of the IEEE Alexandria Subsection from 1995 to 1996. He has also served as a technical referee for Proceedings of the Institution of Electrical Engineers, the IEEE TRANSACTIONS ON COMMUNICATIONS, the IEEE TRANSACTIONS ON INFORMATION THEORY, the IEEE JOURNAL ON SELECTED AREAS IN COMMUNICATIONS, and the IEEE/OSA JOURNAL OF LIGHTWAVE TECHNOLOGY. He is listed in the 14th edition of Marquis Who's Who in the World, 1997. 